\title{
Large nonlocality in macroscopic Hall bars made of epitaxial graphene
}

\author{
A. Nachawaty, ${ }^{1,2}$ M. Yang, ${ }^{3}$ S. Nanot, ${ }^{1}$ D. Kazazis, ${ }^{4,5}$ R. Yakimova, ${ }^{6}$ W. Escoffier, ${ }^{3}$ and B. Jouault ${ }^{1}$ \\ ${ }^{1}$ Laboratoire Charles Coulomb (L2C), UMR 5221, CNRS-Université de Montpellier, 34095 Montpellier, France \\ ${ }^{2}$ LPM, EDST, Lebanese University, Tripoli, Lebanon \\ ${ }^{3}$ LNCMI, Université de Toulouse, CNRS, INSA, Toulouse, France \\ ${ }^{4}$ Centre de Nanosciences et de Nanotechnologies, CNRS, Université Paris-Sud, Université Paris-Saclay, \\ C2N Marcoussis, 91460 Marcoussis, France \\ ${ }^{5}$ Laboratory for Micro and Nanotechnology, Paul Scherrer Institute, 5232 Villigen-PSI, Switzerland \\ ${ }^{6}$ Department of Physics, Chemistry, and Biology, Linköping University, SE-58183 Linköping, Sweden
}

(Received 3 April 2018; revised manuscript received 22 May 2018; published 3 July 2018)

\begin{abstract}
We report on nonlocal transport in large-scale epitaxial graphene on silicon carbide under an applied external magnetic field. The nonlocality is related to the emergence of the quantum Hall regime and persists up to the millimeter scale. The nonlocal resistance reaches values comparable to the local (Hall and longitudinal) resistances. At moderate magnetic fields, it is almost independent on the in-plane component of the magnetic field, which suggests that spin currents are not at play. The nonlocality cannot be explained by thermoelectric effects without assuming extraordinary large Nernst and Ettingshausen coefficients. A model based on counterpropagating edge states backscattered by the bulk reproduces quite well the experimental data.
\end{abstract}

DOI: 10.1103/PhysRevB.98.045403

\section{INTRODUCTION}

For electronic devices, nonlocal measurements refer to the appearance of voltages across contacts which are far away from the expected path of the charge current. Nonlocality is remarkably useful to unveil various phenomena which would be obfuscated by Ohmic contributions in a local configuration, with voltages measured along the charge flow. Nonlocal voltages appear, for instance, in small mesoscopic devices, when Ohm's law breaks down and phase interference comes into play [1]. Nonlocal measurements are also the cornerstone of devices like spin valves, where the signal between excitation and detection is mediated by a neutral spin flow. Nonlocality can also be linked to strong distortion of the charge current paths, as it happens in the quantum Hall effect (QHE) regime, when a quantizing magnetic field forces the charge current to flow preferentially along the device edges [2].

The remarkable electronic properties of graphene were discovered a bit more than 10 years ago [3] and nonlocal measurements revealed them to be a powerful tool to use and study the properties of this new material. Spin phenomena in graphene are of great interest, especially because the small intrinsic spin-orbit coupling favors very long spin relaxation times [4]. Various spin valve devices [5-7] have been tailored to take advantage of this. Nonlocality in graphene was also studied in Hall bar geometries, without the presence of any magnetic element and even without magnetic field. A strong nonlocality was often detected close to the charge neutrality point (CNP), whose origin is still a matter of debate [8]. The nonlocal signal has been attributed to the spin Hall effect (SHE) [9,10], thermo(magneto)electric effects [11], valley currents $[12,13]$, or even to some unknown physical phenomena [14].

In this paper, we are mainly interested in another type of giant nonlocality which is observed in graphene in the QHE regime close to the charge neutrality point (corresponding to a filling factor $v=0$ ). The nonlocality was originally attributed to the existence of a spin current, triggered by the imbalance of Hall resistivities for spin up and down due to Zeeman splitting [15]. Later, additional experimental evidences demonstrated that this nonlocality is not only due to this so-called Zeeman spin Hall effect (ZSHE) and that other mechanisms come into play. First, thermal effects also give rise to a strong nonlocal current. At the excitation point, heat flow is induced by the Ettingshausen effect, perpendicular to the charge flow. The associated thermal gradient produces in turn a nonlocal voltage via the Nernst effect $[11,16]$. Second, theory does not predict counterpropagating edge states to be present at the CNP in the QHE (except for very clean samples); nevertheless, assuming their ad hoc existence allows reproducing quite well several experimental results, either in graphene $[17,18]$ or in two-dimensional $\mathrm{HgCdTe}$-based quantum wells [19], which have a very small gap and a band structure quite similar to graphene.

It is well established that graphene properties are strongly influenced by the substrate. To date, nonlocal properties of epitaxial graphene on silicon carbide (G/SiC), under magnetic fields close to the CNP, have not been reported. The main objective of this paper is to fill this gap, taking advantage of the distinct characteristics of the G/SiC devices: the mobility is low (which prevents the unnecessary complication of an interaction-induced gap opening at $v=0$ ), the spatial homogeneity is good (allowing macroscopic devices at the millimeter scale, with well-controlled and reproduced geometries), and the QHE is remarkably robust (allowing $\mathrm{G} / \mathrm{SiC}$ devices to be used as electrical resistance standards [20,21]).

We studied nonlocality in $\mathrm{G} / \mathrm{SiC}$ by varying the sample size, the temperature, and by applying a magnetic field with a controlled orientation. We found systematically extremely 
large and unexpected nonlocal voltages. Our findings rule out ZSHE as the main origin of the nonlocality. On the contrary, an extended version of the model of McEuen et al. [2], which takes into account both edge and bulk conductivity, describes our results surprisingly well, qualitatively and semiquantitatively.

\section{SAMPLE PREPARATION AND METHODS}

The $\mathrm{G} / \mathrm{SiC}$ were grown epitaxially on the Si-terminated face of semi-insulating $4 \mathrm{H}-\mathrm{SiC}$ at high temperature, $T=2000 \mathrm{~K}$. Hall bars of various sizes were patterned on $\mathrm{G} / \mathrm{SiC}$ using standard electron-beam lithography. The samples were then encapsulated in a bilayer of resists as described in Ref. [22]. The resists can be used to lower the Fermi level closer to the Dirac point using either UV illumination [23] or the corona discharge method [24].

Local and nonlocal magnetotransport measurements were made on four G/SiC Hall bars, called G13, G14, G21, and G34. The samples have a length of $420 \mu \mathrm{m}$ and a width of $100 \mu \mathrm{m}$, except for G34, which has a length of $100 \mu \mathrm{m}$ and a width of $20 \mu \mathrm{m}$. The Hall bars G13, G14, and G21 originate from the same graphene growth. The Hall bar G34 comes from another growth. The angle of the $\mathrm{SiC}$ steps with respect to the Hall bar axis was also carefully checked. This angle is about $30^{\circ}$ for $\mathrm{G} 13, \mathrm{G} 14$, and $\mathrm{G} 21$, and $0^{\circ}$ for $\mathrm{G} 34$.

Each sample was prepared close to the charge neutrality point at room temperature using the corona method as described in our previous work [22]. Afterwards, each sample was cooled down to low temperature $T=1.7 \mathrm{~K}$ several times (up to 13 times for G14) in order to assess the reproducibility of the nonlocality and to vary the residual doping. At low temperatures, all prepared samples had low Hall carrier densities $n_{H}$ with values in the range $n_{H}=-(0.9-3) \times 10^{10} \mathrm{~cm}^{-2}$, corresponding to a Fermi energy $E_{F} \simeq-20 \mathrm{meV}$ below the CNP. This ensures that the Fermi energy resides in the electronhole puddles, as the potential fluctuation in $\mathrm{G} / \mathrm{SiC}$ is of the order of a few tens of meV [25].

In the following, we define the resistance $R_{i j, k l}=V_{k l} / I_{i j}$, where $V_{k l}$ is the ac voltage drop between contacts $k$ and $l$ measured by lock-in technique, and $I_{i j}$ is a low-frequency $(\sim 10 \mathrm{~Hz})$ ac current biased between contacts $i$ and $j$.

\section{RESULTS}

\section{A. Local measurements}

For all samples, we label the contacts as reported in the inset of Fig. 1(a). Figure 1(a) shows the transverse and longitudinal magnetoresistances for sample G34. We observe that under magnetic field, the Hall resistance changes sign when the magnetic field $B$ increases, evolving from negative to positive values (from holes to electrons). Furthermore, an unexpected bump is observed in the longitudinal resistance at $B \simeq 2 \mathrm{~T}$, approximately at the magnetic field for which the Hall resistance cancels.

This remarkable behavior was recently reported by our group [22] in similar samples. The evolution of the magnetoresistances cannot be explained by a standard two-fluid Drude model. Charge transfer as a function of magnetic field is almost systematically observed at high fields in $\mathrm{G} / \mathrm{SiC}$ samples, because of the quantum capacitive coupling between graphene

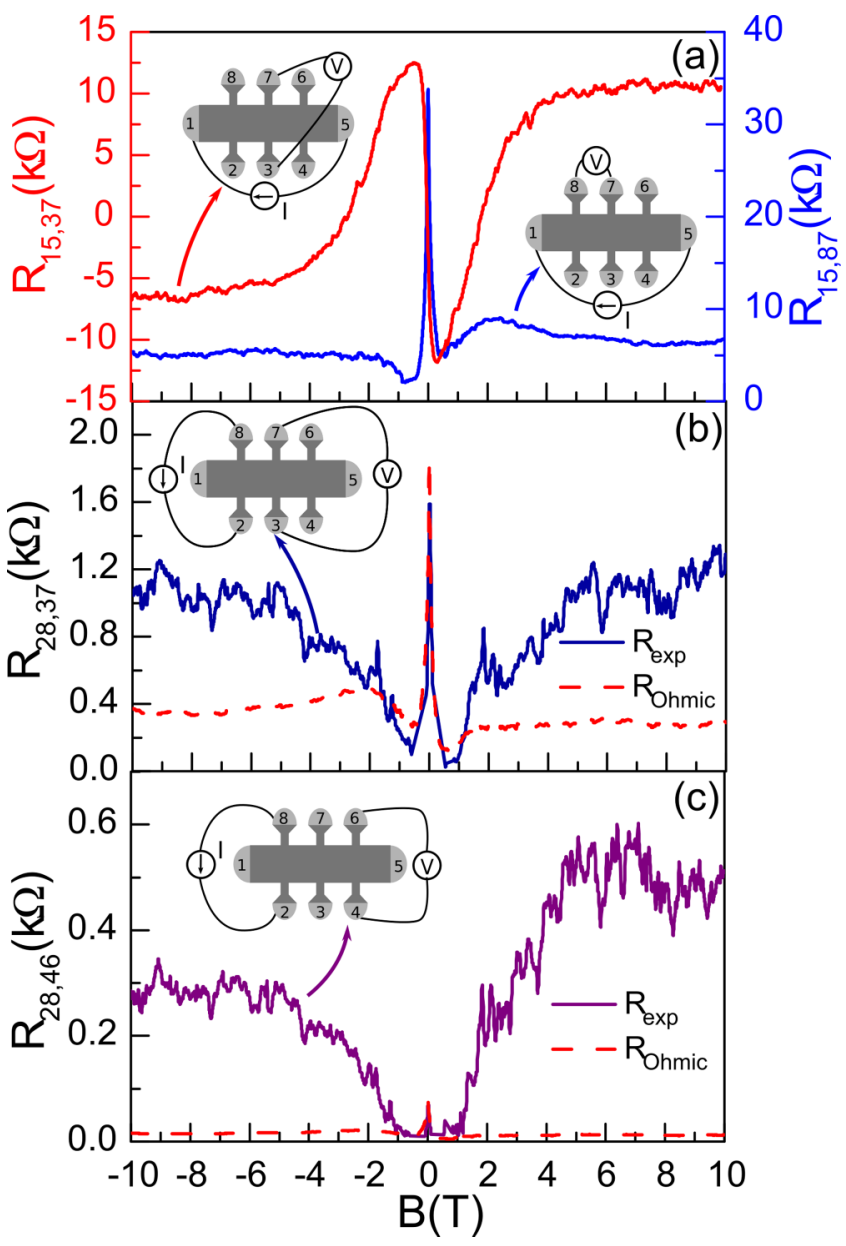

FIG. 1. Local and nonlocal measurements for sample G34. (a) Transverse and (b) longitudinal magnetoresistances at $T=1.7 \mathrm{~K}$ and $I=10 \mathrm{nA}$. (b), (c) Nonlocal resistances $R_{28,37}$ and $R_{28,46}$ as a function of magnetic field. The red dashed lines represent the nonlocal resistance calculated from the Ohmic contribution law, see main text. The inset in (a) is an optical image of one of the largest samples (Hall bar width $100 \mu \mathrm{m}$, Hall bar length $420 \mu \mathrm{m}$ ) where the labeling of the contacts, as used in the main text, is reported.

and the so-called "dead graphene layer," as explained by the model developed in Ref. [26]. However, this model, in its original form, cannot explain the data observed in Fig. 1(a), as the cancellation of the Hall resistance and the bump of the longitudinal resistance occur at far too small magnetic field. The data suggest that the Fermi energy increases with $B$ in such a way that the Fermi level evolves from the bottom to the top of the electron-hole puddles.

The exact relation between $B$ and $E_{F}$ depends on disorder and cannot be established precisely. In the following, we do not discuss its origin. By contrast, we focus on the remarkable evolution of the nonlocal voltages in the Hall bars prepared close to the CNP in the presence of a magnetic field.

\section{B. Nonlocal measurement}

Figure 1(b) shows nonlocal resistance $R_{28,37}$ as a function of magnetic field at $T=1.7 \mathrm{~K}$. The solid line represents the experimental data. The nonlocal resistance quickly drops 
at very low magnetic field and then starts increasing. The nonlocal resistance reaches values of the order of $1 \mathrm{k} \Omega$. This value saturates at higher magnetic fields corresponding to the electron-doped region. The nonlocal resistance $R_{28,46}$ [Fig. 1(c)], further away from the current injection contacts, follows a similar field dependence and reaches also a rather large value of $\simeq 500 \Omega$ above $B=2 \mathrm{~T}$.

Similar trends have been observed for the three other samples, as shown, for instance, in Fig. 2 (solid lines) for the Hall bar G14. In this case, the nonlocal resistances $R_{46,37}$ and $R_{46,28}$ reach higher values $(10 \mathrm{k} \Omega)$ than in the Hall bar G34, even if the Hall bar G14 is about 4 times larger.

\section{Semiclassical current spreading}

The first contribution to consider is how the classical spreading of the charge flow inside the Hall bar gives rise to a nonlocal resistance. It follows the equation [27]

$$
R_{\text {Ohmic }}=\frac{4}{\pi} \rho_{x x} \exp (-\pi L / W),
$$

where $\rho_{x x}$ is the resistivity, $L$ is the distance separating the current injection from the voltage detection, and $W$ is the Hall bar width.

The dashed lines in Figs. 1(b) and 1(c) correspond to the nonlocal resistance calculated using the Ohmic contribution law, Eq. (1), where the resistivity is estimated from the local resistance: $\rho_{x x} \simeq R_{15,87}$. The geometric factor is $L / W=1$ for $R_{28,37}$ and $L / W=2$ for $R_{28,46}$. The Ohmic contribution corresponds well to the measured nonlocal resistances at $B=$ $0 \mathrm{~T}$ and its drop at very low field $|B|<1 \mathrm{~T}$. However, Eq. (1) fails to explain how the nonlocal resistances increase at higher $B$ and rapidly underestimate them.

\section{Zeeman spin Hall effect}

Nonlocal resistances are often explained by spin diffusion: when a charge current flows, spin currents are created transversally via spin Hall effect. These spin currents induce a nonlocal voltage outside the charge current path detected via the inverse spin Hall effect. This charge/spin coupling originates from intrinsic spin-orbit coupling or extrinsic effects in the case of graphene. The spin-induced nonlocal resistance is given by [28]

$$
R_{\mathrm{NL}}=\rho_{x x} \frac{W}{8 \lambda_{s}} \theta_{\mathrm{SH}}^{2} \exp \left(-L / \lambda_{s}\right),
$$

where $\theta_{\mathrm{SH}}$ is the Hall spin angle and $\lambda_{s}$ is the spin diffusion length.

The observed nonlocal resistance can be traced as a function of $L$ for different samples and fitted with Eq. (2) as a function of $\theta_{\mathrm{SH}}$ and $\lambda_{s}$. We could not find any reasonable fit of Eq. (2) from any of the data. In particular, the nonlocal resistance was much smaller (up to ten times) in the smallest sample ( $L=20 \mu \mathrm{m}$, Fig. 1) than in the largest ones $(L=100 \mu \mathrm{m}$, Fig. 2). And for most of our measurements, see Appendix B, Eq. (2) gives much larger spin diffusion length $\lambda_{s}>200 \mu \mathrm{m}$ than what is reported in the literature [6]. Therefore, the observed nonlocal resistance does not seem to originate from spin currents.

Equation (2) was introduced in Ref. [28], where SHE was of spin-orbital origin. However, SHE may have several
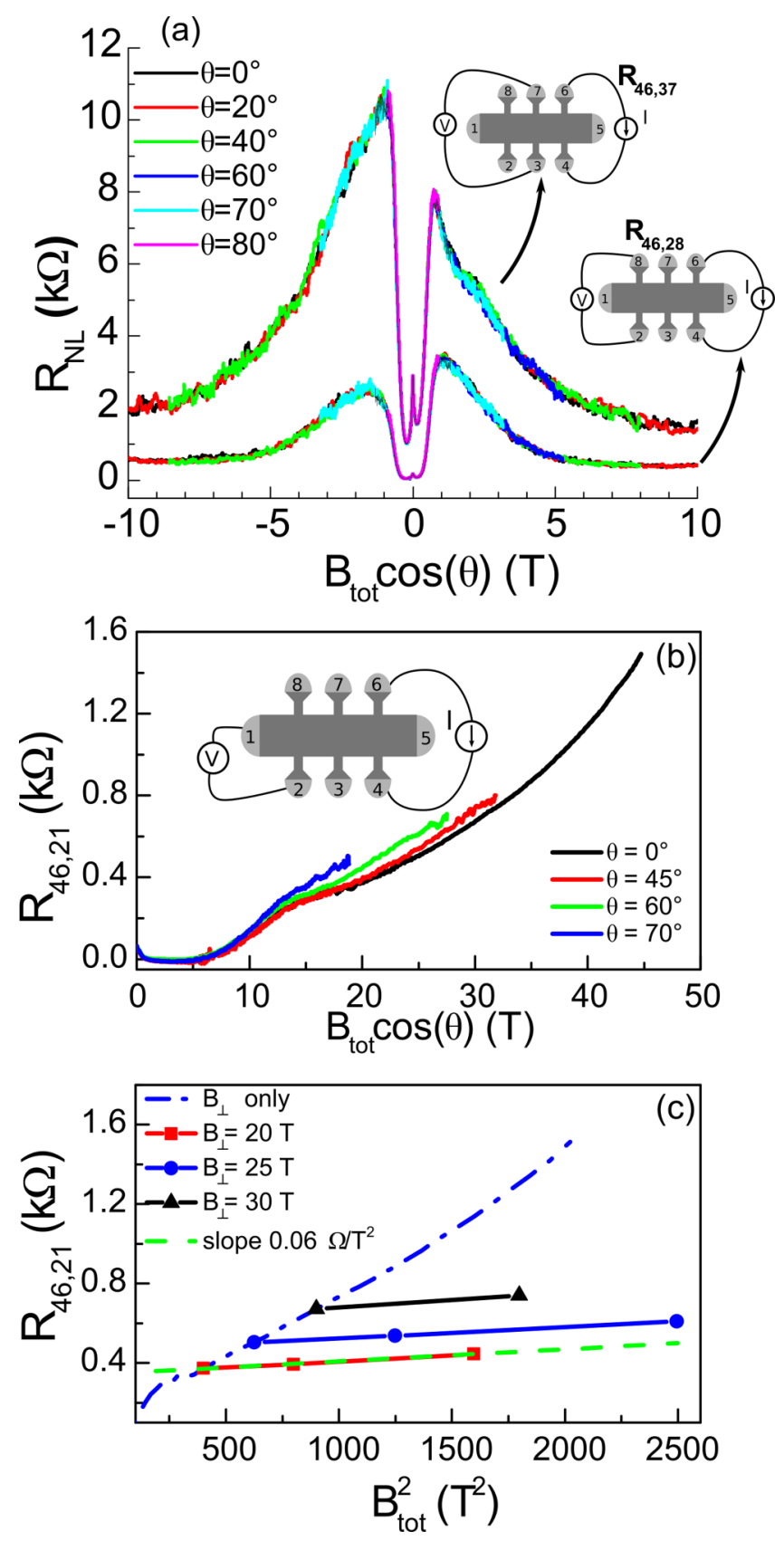

FIG. 2. (a) Nonlocal resistances $R_{46,37}$ and $R_{46,28}$ as a function of the perpendicular component of the magnetic field for several tilt angles, measured at $T=1.7 \mathrm{~K}$ and $I=10 \mathrm{nA}$ on sample G14. (b) Evolution of the nonlocal resistance $R_{46,21}$ for sample G21, at $T=1.3 \mathrm{~K}$ and $I=10 \mu \mathrm{A}$, with a total magnetic field $B_{\text {tot }}$ set at an angle $\theta$ with respect to the sample plane. (b) Dependence of $R_{46,21}$ on squared total magnetic field $B_{\text {tot }}^{2}$ for different fixed out-of-plane magnetic fields $B_{\perp}=20,25$, and $30 \mathrm{~T}$. The green dashed line fits the quadratic dependence on the total magnetic field: $R_{46,21}^{\mathrm{NL}}=R_{0}+\beta B_{\mathrm{tot}}^{2}$ for $B_{\perp}=20 \mathrm{~T}$, where $\beta=0.06 \Omega / \mathrm{T}^{2}$ and $R_{0}=350 \Omega$. For $B_{\perp}=25$ and $30 \mathrm{~T}$, the same quadratic dependence is found.

extrinsic origins and Eq. (2) will be still valid. Let us assume in the following that SHE is induced by interplay between the Zeeman interaction and magnetotransport. This effect, called the Zeeman spin Hall effect $[15,29]$, is maximized near the 
charge neutrality point. The magnetic field splits the Dirac cone via Zeeman effect and generates electron- and holelike carriers with opposite spins. The Hall angle is then given by [15]

$$
\theta_{\mathrm{SH}}=\frac{1}{2 \rho_{x x}} \frac{\partial \rho_{x y}}{\partial \mu} E_{z}
$$

where $\mu$ is the chemical potential and $E_{Z}$ is the Zeeman splitting. As ZSHE does not impact the validity of Eq. (2), introducing Eq. (3) into Eq. (2) predicts a nonlocal resistance for ZSHE proportional to

$$
R_{\mathrm{NL}} \propto \frac{1}{\rho_{x x}}\left(\frac{\partial \rho_{x y}}{\partial \mu} E_{Z}\right)^{2} .
$$

The longitudinal and Hall resistances depend mainly on the out-of-plane component of the magnetic field $B_{\perp}$, whereas $E_{Z}$ is proportional to the total magnetic field $B_{\text {tot }}$. So Eq. (4) can be rewritten as

$$
R_{\mathrm{NL}}=\beta\left(B_{\perp}\right) B_{\mathrm{tot}}^{2},
$$

where $\beta$ is a function which depends only on $B_{\perp}$. The relation was first noticed in Ref. [11] and can be used to check the part of the ZSHE contribution to $R_{\mathrm{NL}}$.

Figure 2(a) plots the nonlocal resistances $R_{46,37}$ and $R_{46,28}$ as a function of the out-of-plane component of the magnetic field, $B_{\perp}=B_{\text {tot }} \cos \theta$, for several tilt angles $\theta$, for sample $G 14$. It is clear that the nonlocal resistances $R_{46,37}\left(B_{\perp}\right)$ and $R_{46,28}\left(B_{\perp}\right)$ do not depend on the in-plane component $B_{\|}$of the magnetic field and Eq. (5) cannot fit the results.

Nevertheless, the square factor $B_{\text {tot }}^{2}$ in Eq. (5) could become dominant at high enough magnetic fields. In order to test this prediction, we measured the local and nonlocal resistances for another sample, G21, at very large $B$. The nonlocal resistance $R_{46,21}$ is shown in Fig. 2(b) as a function of a pulsed magnetic field for different tilt angles. There is a weak dependence of the nonlocal resistance on the in-plane magnetic field which appears at magnetic fields higher than $10 \mathrm{~T}$. In Fig. 2(c) we replotted $R_{46,21}$ as a function of $B_{\text {tot }}^{2}$ for $B_{\perp}=20,25$, and $30 \mathrm{~T}$. The data can be fitted by Eq. (5), assuming there is an additional constant term: $R_{46,21}=R_{0}+\beta B_{\text {tot }}^{2}$. The slope $\beta$, equal to $0.06 \Omega / \mathrm{T}^{2}$, is slightly smaller (by a factor of 2) than the values reported in Ref. [11].

Therefore, we could identify a part of the nonlocal resistance which obeys Eq. (5) and which we attribute to ZSHE. However, the main part of the nonlocal resistance $\left(R_{0}\right)$ does not depend on $B_{\|}$and must have another origin.

\section{E. Thermoelectric effects}

A thermal origin for nonlocal voltages was proposed in Ref. [11]. In the presence of a transverse magnetic field, the Ettingshausen effect [30] and Joule heating generate a heat flow perpendicular to the injected current. This induces a thermal gradient $\partial T / \partial x$ which propagates into the Hall bar and is converted into nonlocal voltages by the Nernst effect, which is quantified by the Nernst coefficient [30] $S_{y x}=E_{y}(\partial T / \partial x)$.

Joule heating and the Ettingshausen effect have very distinct experimental signatures. Joule heating induces a temperature gradient proportional to the heating power, $\partial T / \partial x \propto Q_{\text {Joule }}=$ $R I^{2}$. Because of this quadratic dependence on the current,

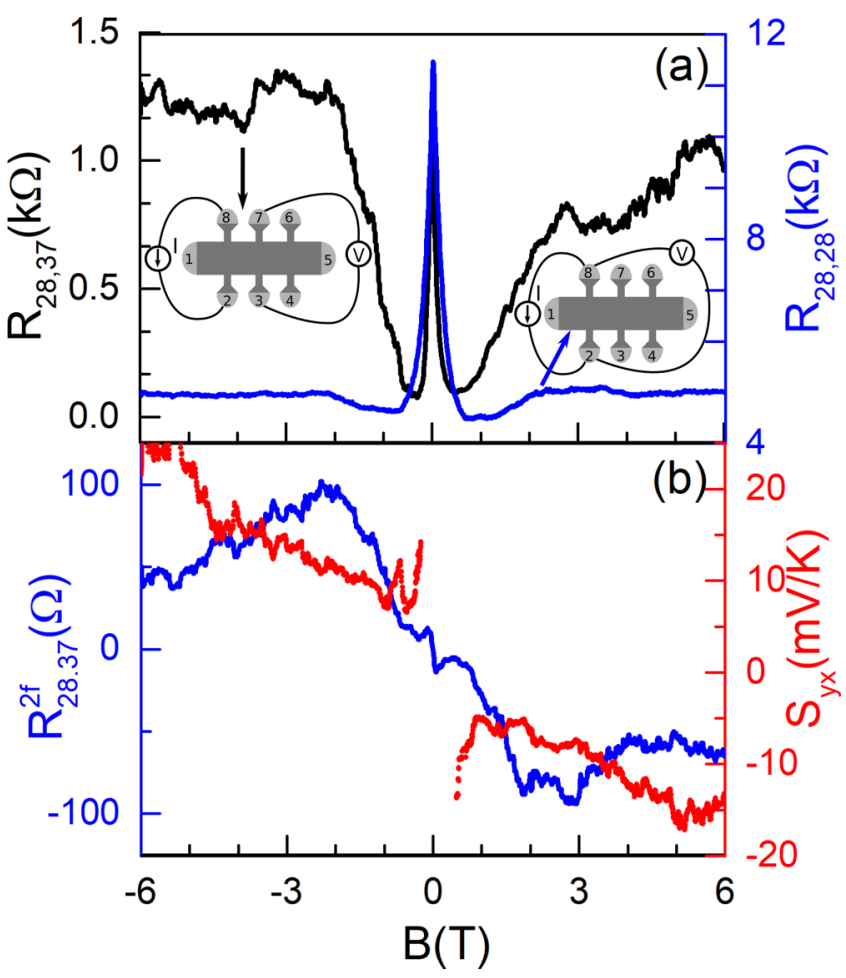

FIG. 3. (a) Nonlocal magnetoresistance $R_{28,37}$ measured on sample G34 at $T=1.7 \mathrm{~K}$ and $I=1 \mu$ A (black line). The two-contact resistance $R_{28,28}$ (blue line) used to estimate $Q_{\text {Joule }}$ is also shown. (b) Measured second harmonic resistance $R_{28,37}^{2 f}$ (blue line) and corresponding estimation of the Nernst coefficient $S_{y x}$ following Eq. (6).

Joule heating can only appear in the second harmonic of the nonlocal resistance, $R_{\mathrm{TE}}^{2 f}$. By contrast, the Ettingshausen effect is proportional to the current, $\partial T / \partial x \propto Q_{E t t} \propto T S_{y x} I$, and appears directly in the first harmonic of the nonlocal resistance, $R_{\mathrm{TE}}$.

It is then possible to show that Ettingshausen and Joule signals are proportional, which allows extracting a value of the Nernst coefficient [11]:

$$
S_{y x}=\frac{\gamma Q_{\mathrm{Joule}}}{T I} \frac{R_{\mathrm{TE}}}{R_{\mathrm{TE}}^{2 f}},
$$

where $\gamma$ is a constant which depends on the sample geometry.

Figure 3(a) shows the first harmonic of the nonlocal resistance $R_{28,37}$ measured in sample G34 at $I_{28}=1 \mu \mathrm{A}, T=$ $1.7 \mathrm{~K}$, and low frequency $f \simeq 10 \mathrm{~Hz}$. The Ohmic contribution $R_{2 c}=R_{28,28}$, used to estimate the Joule heating, is also shown. The second harmonic resistance $R_{28,37}^{2 f}$ is reported in Fig. 3(b). The same figure also shows $S_{y x}(B)$ calculated using Eq. (6). From the Hall bar geometry we estimate $\gamma \simeq 0.3$. The Nernst coefficient appears to be asymmetric with $B$, as expected. However, $S_{y x}$ also reaches unrealistically high values of more than $S_{y x} \simeq 20 \mathrm{mV} / \mathrm{K}$ at $|B| \simeq 6 \mathrm{~T}$. This is 100 times larger than values typically reported at the charge neutrality point in graphene [31-33]. This suggests that the nonlocality does not originate from thermal effects. It is also worth noting that the $2 f$ signal was unmeasurable at currents lower than $1 \mu \mathrm{A}$. This 


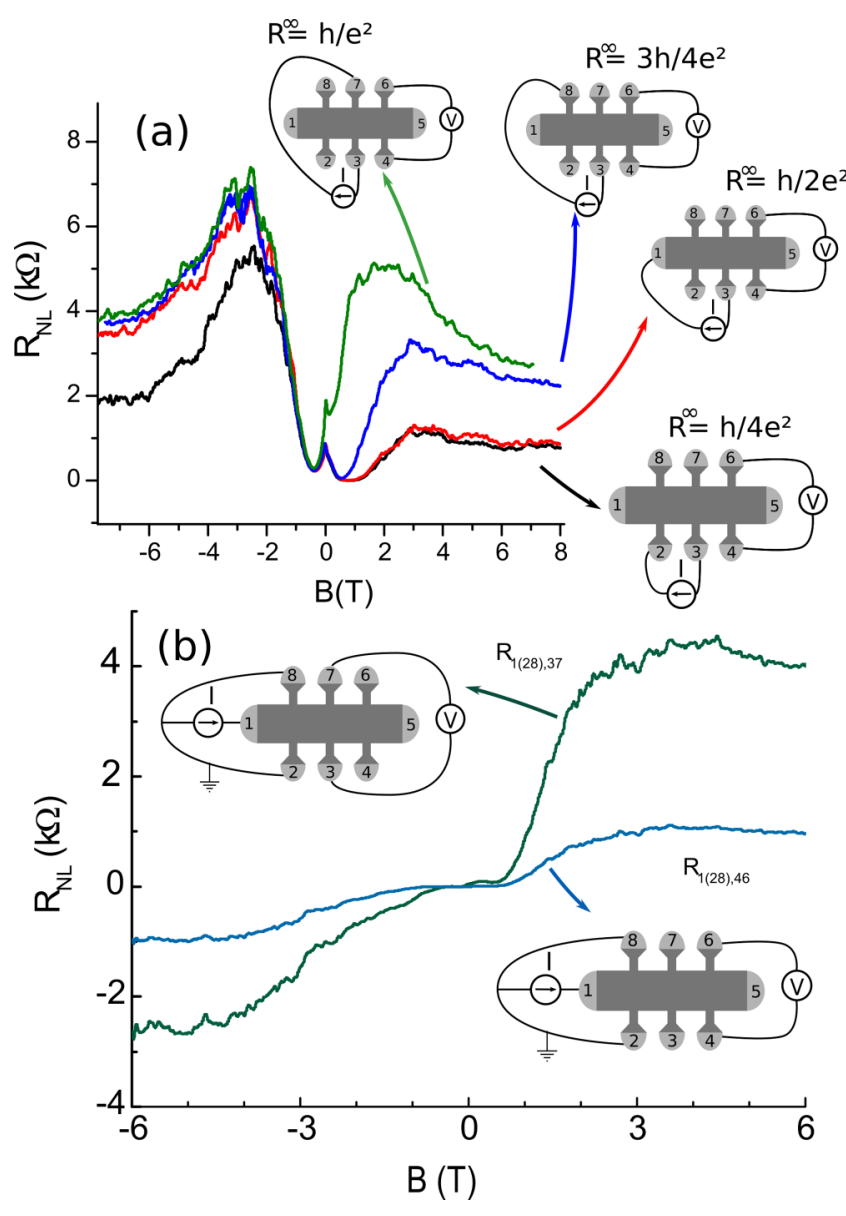

FIG. 4. (a) Magnetoresistances $R_{23,64}, R_{13,64}, R_{83,64}$, and $R_{73,64}$ for the Hall bar G14, at $T=1.7 \mathrm{~K}$ and $I=10 \mathrm{nA}$. The resistances $R^{\infty}$ expected for perfectly transmitted counterpropagating edge states are also reported for the four configurations. (b) Magnetoresistances $R_{1(28), 46}$ and $R_{1(28), 37}$ for the same Hall bar G14, under the same experimental conditions.

indicates that the thermoelectric contribution is weak for the typically used currents of $10 \mathrm{nA}$.

\section{F. Counterpropagating edge states}

So far, the role of edge states when Landau levels are formed has not been mentioned. Nevertheless, their existence has been assumed in several similar experiments to explain the nonlocality [17-19]. It was also clearly observed that in clean samples, counterpropagating edge states are formed and lead to an insulating state under perpendicular magnetic field, which can start exhibiting well-quantized values when the relative spin contribution is increased under a tilted magnetic field [34]. Besides, a recent theory predicts the existence of additional edge states in $\mathrm{G} / \mathrm{SiC}$ at low filling factors [35]. These edge states appear because the electrostatic potential imposes a charge modulation at the sample edge [36].

Figure 4(a) shows four different nonlocal resistances $R_{i 3,64}$ measured in Hall bar G14 for a given corona preparation, where only the current injection contact, $i$, changes. We find that the resistance increases progressively in the following order: $R_{23,64}, R_{13,64}, R_{83,64}$, and $R_{73,64}$. From the Landauer-Büttiker formalism, one gets an estimate for these resistances, assuming that there is an infinite mean-free path for backscattering between the two edge states: $R_{23,64}^{\infty}=h / 4 e^{2}, R_{13,64}^{\infty}=h / 2 e^{2}$, $R_{83,64}^{\infty}=3 h / 4 e^{2}$, and $R_{73,64}^{\infty}=h / e^{2}$. Thus, the resistance variation from one configuration to the other is in qualitative agreement with the edge states model.

However, this estimate quantitatively gives only the correct order of magnitude for the resistances. A strong $\pm B$ asymmetry is also noticeable. An interesting configuration consists of injecting current from contact 1 , grounding both contacts 2 and 8 , while the nonlocal resistances $R_{1(28), 37}$ and $R_{1(28), 46}$ are measured between contact pairs $(3,7)$ and $(4,6)$, respectively. In such a configuration, the Ettingshausen effect induces a heat flow perpendicular to the main Hall bar axis. Similarly, ZSHE induces a spin current which is also perpendicular to the Hall bar axis. Moreover, if edge states are responsible for the nonlocality and circulate around the sample, then they should impose all contacts 3-7 to have the same null potential. Therefore, in all cases, one expects $R_{1(28), 37}=R_{1(28), 46}=0$.

Figure 4(b) shows the magnetoresistances $R_{1(28), 37}$ and $R_{1(28), 46}$ for sample G14. Surprisingly, these two resistances, again, become large when $B$ increases. Also, they are mainly antisymmetric with $B$, while ZSHE and thermal effects are symmetric in $B$. This confirms that both ZSHE and the thermal effect are of little importance in the appearance of nonlocal resistances. Moreover, this experiment demonstrates that bulk conductivity plays a role in the appearance of the nonlocality and an edge-to-bulk leakage is present.

\section{SUMMARY AND CONCLUSION}

The main results of this work are summarized in Fig. 5, which reports the maxima of the nonlocal resistances $R_{28,37}^{\max }$ $(L / W=1)$ and $R_{28,46}^{\max }(L / W=2)$ for three different Hall bars and various corona preparations. The maxima corresponding to the same Hall bar and the same preparation are linked by a dotted line. These data can be compared with four models. The solid blue line corresponds to the nonlocal resistance given the deviation of the current flow, as given by Eq. (1) with $\rho_{x x}=10 \mathrm{k} \Omega$, the typical transverse resistance measured in the devices. The orange line corresponds to the spin diffusion model and is given by Eq. (2), assuming unrealistically high values $\theta_{\mathrm{sh}}=1$ and $\lambda=100 \mu \mathrm{m}$ [18]. The thermoelectric contribution is given by the formula $R_{28,37}=$ $(W / w) S_{x y}^{2} T / d \kappa_{x x}$, where $W$ is the Hall bar width, $w$ is the width of the lateral probes $(W / w \simeq 5), d=0.3 \mathrm{~nm}$ is the graphene thickness, $\kappa_{x x}$ is the thermal conductivity, and $S_{x y}$ is the Nernst coefficient. The second nonlocal resistance, located further away from the injection point, can be estimated as a fraction of $R_{28,37}: R_{28,46} \simeq R_{28,37 / 3}$ [11]. From the literature [31,37], taking $\kappa_{x x}=1 \mathrm{Wm}^{-1} \mathrm{~K}^{-1}$ and $S_{x y}=100 \mu \mathrm{V} / \mathrm{K}$, we get the estimate indicated by the red solid line in Fig. 5. These three models severely underestimate the observed nonlocal resistances. At the contrary, assuming perfectly transmitted counterpropagating edge states in the Hall bars, we obtain the purple line in Fig. 5, which overestimates the experimental nonlocal resistances. An even better agreement with the data can be obtained by adapting the model of McEuen et al. [2] to the graphene case (see Appendix A). The model includes indeed another parameter, $\rho_{0}$, which describes the edge-to-bulk 

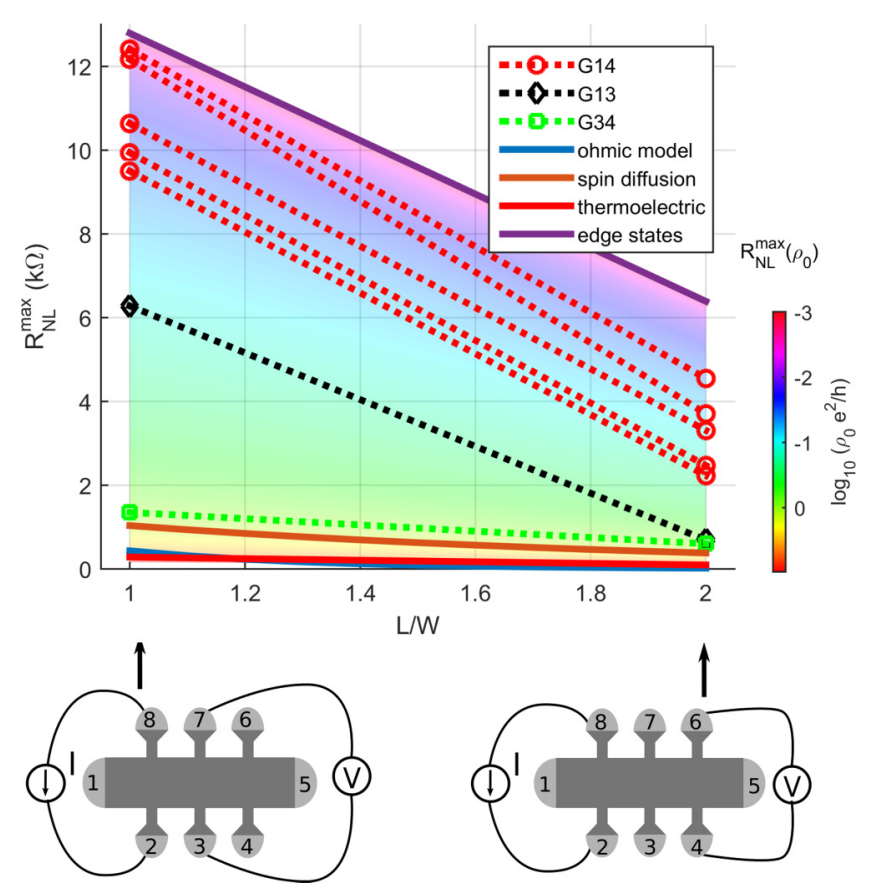

FIG. 5. (a) Open symbols: maxima of the nonlocal magnetoresistances $R_{28,37}^{\max }(L / W=1)$ and $R_{28,46}^{\max }(L / W=2)$, measured at $T=1.7 \mathrm{~K}$ in the interval $-13 \mathrm{~T}$ to $13 \mathrm{~T}$ (excluding the $B=0 \mathrm{~T}$ peak). The dotted lines link maxima corresponding to the same Hall bar and the same corona preparation. Red dotted lines: Hall bar G14 (five different corona preparations); black dotted line: Hall bar G13; green dotted line: Hall bar G34. The solid lines are given by the models of current flow (blue line), spin diffusion (orange line), thermoelectric model (red line), and perfectly transmitted edge states (purple line). The color map shows the edge-to-bulk leakage parameter $\rho_{0}$ as a function of the calculated maxima of nonlocal resistances, following the model presented in Appendix A.

leakage when the Fermi energy is exactly at the CNP. The color map in Fig. 5 shows the evolution of the maximum of the nonlocal resistances, $R_{\mathrm{NL}}^{\max }\left(\rho_{0}\right)$, when $\rho_{0}$ is varied. For small values of $\rho_{0}\left(\rho_{0}=10^{-3} \mathrm{~h} / \mathrm{e}^{2}\right)$, the nonlocal resistances correspond to what is expected for perfectly transmitted edge states (purple line). As $\rho_{0}$ increases, the bulk shunts the edge current and the nonlocal resistances decrease. From the figure, we can get a rough estimate for $\rho_{0}$. For the Hall bars G14, G13, and G34 we obtain $\log _{10}\left(\rho_{0} e^{2} / h\right)=(-1.4 \pm 0.5),(-0.5 \pm 0.6)$, and $(0 \pm 0.02)$ respectively. However, these values reflect only poorly the real edge-to-bulk leakage, as a complete treatment should take into account backscattering between the edge states.

To conclude, we have investigated the local and the nonlocal voltages appearing in epitaxial graphene Hall bars close to the CNP. Very large nonlocal resistances are observed systematically when the QHE regime takes place. In some cases, the nonlocal resistances are so large that they approach $h / 2 e^{2}$ and can surpass the local resistances. These high resistances are only observed when the samples have been prepared to have the Fermi energy close to the Dirac point at $B=0 \mathrm{~T}$. They also strongly decrease when the current or the temperature increases. A model of edge conduction explains the data qualitatively and semi-quantitatively.
We did not discuss the residual $\pm B$ asymmetry in the local and nonlocal magnetoresistances, which can be seen clearly in most of our measurements, as shown in Figs. 1-4. A similar asymmetry was recently observed and interpreted in Ref. [18] in terms of spin-dependent current at the grain boundaries of chemical-vapor-deposited graphene. The asymmetry could also be reproduced in our model if it is assumed that the conduction (of either edge or bulk states) depends on spin polarization. However, we do not expect such grain boundaries in our $\mathrm{G} / \mathrm{SiC}$ samples, and the in-plane component of the magnetic field has no effect on the nonlocality. As a consequence, this asymmetry needs further analysis.

\section{ACKNOWLEDGMENTS}

This work has been supported in part by the French Agence Nationale de la Recherche (ANR-16-CE09-0016) and through the grant NEXT n ANR-10-LABX-0037 in the framework of the "Programme des Investissements d'Avenir". Part of this work was performed at LNCMI under EMFL proposal TSC06-116.

\section{APPENDIX A}

Let us assume that two counterpropagating edges states are present in the samples and are spin degenerate. We assume that coherence plays no role and that interchannel scattering can be weak, which yields to two independent electrochemical potentials for the two edge states. These edge states coexist with a disordered bulk, as shown in Fig. 6(a). We reuse the model proposed by McEuen et al. [2] in which each segment of the Hall bar is modeled by a barrier which backscatters the edge states through the bulk from one side of the segment to the other, see Figs. 6(b) and 6(c). We neglect interchannel scattering inside graphene. The transmission probability $T_{j}$ of the $j$ th segment can be traced back to an effective resistivity
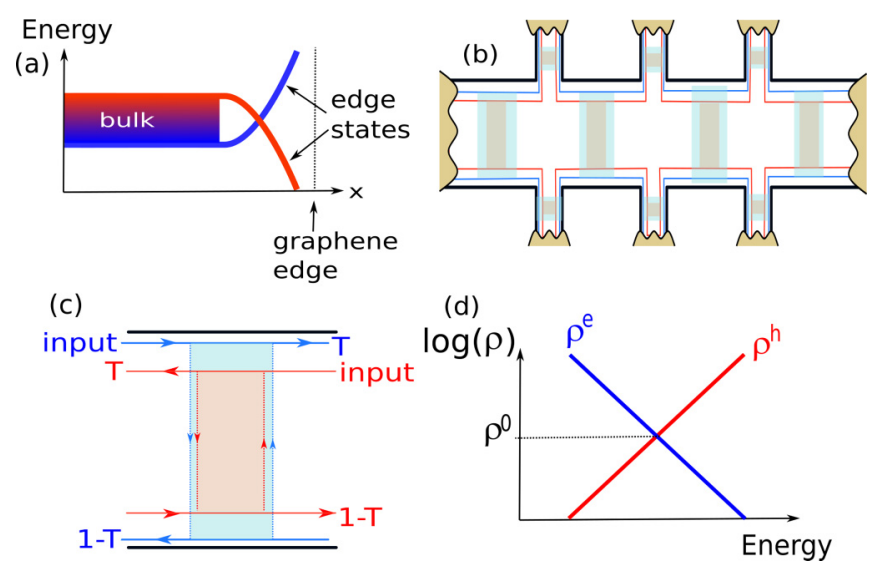

FIG. 6. (a) Simplified scheme of the $N=0$ Landau-level structure, with the disordered bulk and the two spin-degenerate edge states. (b) A model of the device where each segment is replaced by a barrier which backscatters separately the two counterpropagating $N=0$ edge states. (c) Close view of one segment. (d) Energy evolution of the effective resistivities $\rho^{e}$ and $\rho^{h}$ used in the model near the charge neutrality point, $\rho^{e} \rho^{h}=\left(\rho^{0}\right)^{2}$ for simplicity. 


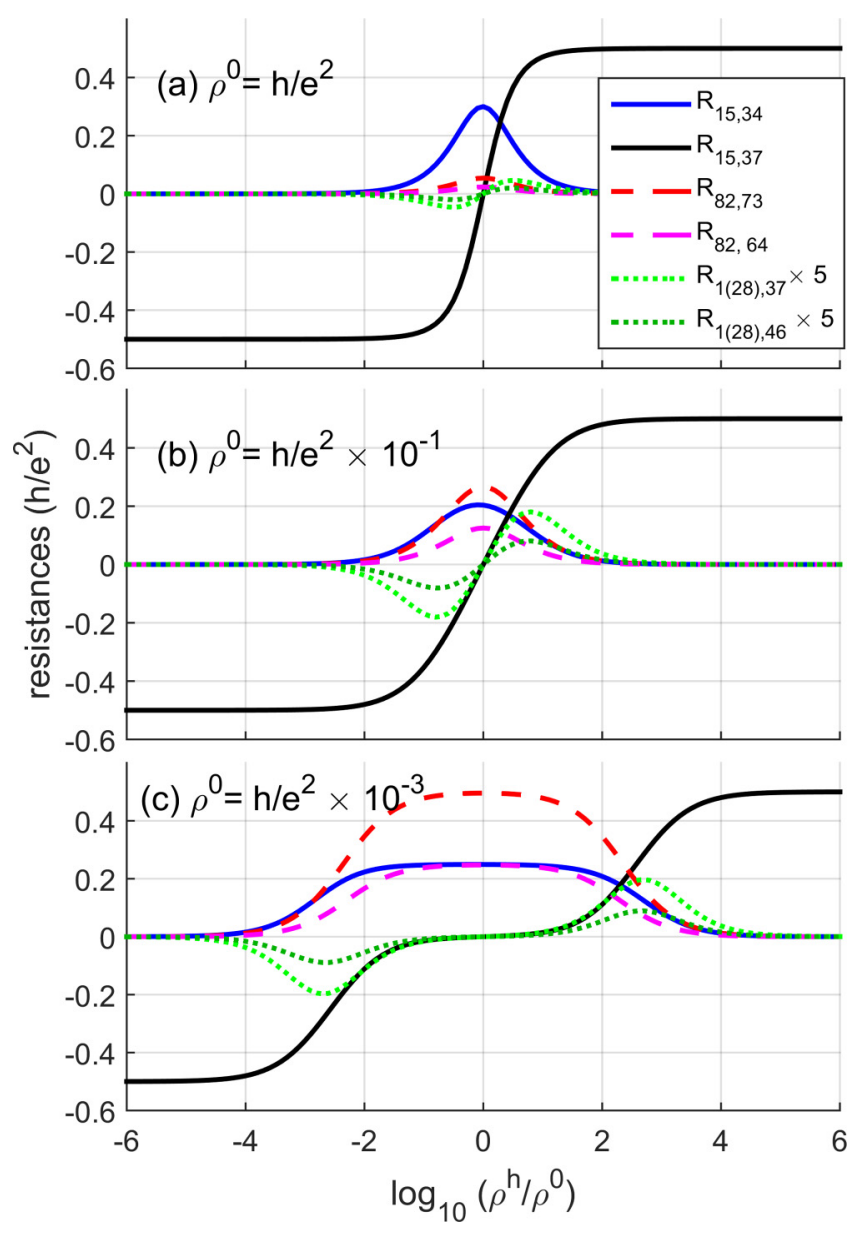

FIG. 7. Evolution of the sample resistances as a function of $\log \left(\rho^{h} / \rho^{0}\right) \propto E_{F}$, from strongly backscattered to almost perfectly transmitted edge states: (a) $\rho^{0}=h / e^{2}$, (b) $\rho^{0}=h / e^{2} \times 10^{-1}$; and (c) $\rho^{0}=h / e^{2} \times 10^{-3}$. The same cases as the experiment in Fig. 4 are plotted: $R_{15,34}$ (longitudinal resistance, solid blue line), $R_{15,37}$ (Hall resistance, solid black line), $R_{82,73}$ and $R_{82,64}$ (nonlocal resistances, dashed red and magenta lines), and $R_{1(28), 37}$ and $R_{1(28), 46}$ (nonlocal resistances, dotted green and dark green lines).

by the equation

$$
\rho^{N}\left(L_{j} / W_{j}\right)=\left(h / e^{2}\right)\left(1-T_{j}\right) / T_{j},
$$

where $N=(e, h)$ labels the edge states, $L_{j}$ and $W_{j}$ are the length and width of the $j$ th segment, and $\rho^{N}$ is the effective resistivity of the $N$ th channel only. When the Fermi energy $E_{F}$ increases from the valence band to the conduction band and scans the vicinity of the charge neutrality point, $\rho^{h}$ increases to infinity and $\rho^{e}$ decreases to zero. Thus, there must be a Fermi energy $E^{0}$, where both effective resistivities are equal: $\rho^{e}\left(E_{F}=E^{0}\right)=\rho^{h}\left(E_{F}=E^{0}\right)=\rho^{0}$. The exact relation between $\rho^{e}$ and $\rho^{h}$ is not known. For the sake of simplicity, we assume that $\rho^{e} \rho^{h}=\left(\rho^{0}\right)^{2}$, as shown in Fig. 6(d).

Then all resistances can be calculated using the LandauerBüttiker formalism $[1,38]$ as a function of only two parameters, $\rho^{h}$ and $\rho^{0}$. Interchannel scattering is taken into account only in the Ohmic contacts. Figure 7 shows several calculated local and nonlocal resistances as a function of $\log \left(\rho^{h} / \rho^{0}\right)$, which increases monotonously with $E_{F}$. The important parameter is
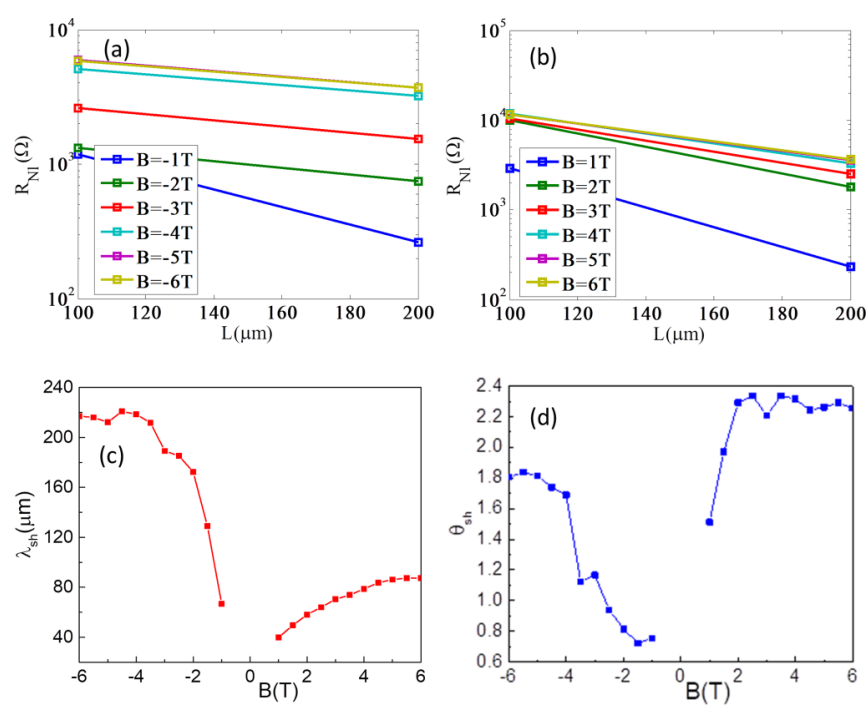

FIG. 8. (a) Nonlocal resistances for G14, at $T=1.7 \mathrm{~K}$ (12th cooldown) and at different fixed negative magnetic fields. (b) Same as (a) but for positive magnetic field. (c) Spin diffusion length, and (d) Hall angle coefficient extracted from the fit using Eq. (2).

$\rho^{0}$, which controls if the edges states are decoupled from the bulk $\left(\rho^{0} \ll h / e^{2}\right)$ or backscattered $\left(\rho^{0} \geqslant h / e^{2}\right)$ at $E_{F}=E^{0}$. At high $\rho^{0}\left[\rho^{0}=h / e^{2}\right.$, panel (a) $]$, all nonlocal resistances are negligible when compared to the local longitudinal resistances. At lower $\rho^{0}\left[\rho^{0}=h / e^{2} \times 10^{-1}\right.$, panel (b) $]$, nonlocal and local resistances become comparable. Finally, at even lower $\rho^{0}\left[\rho^{0}=h / e^{2} \times 10^{-3}\right.$, panel (c) $]$, close to $E_{F}=E^{0}$ backscattering is negligible and all resistances correspond to what is expected for perfectly transmitted edges states. In this last case, the nonlocal resistances close to the CNP can be significantly larger than the local ones.

Interestingly, the model also predicts sizable nonlocal resistances $R_{1(28), 37}$ and $R_{1(28), 46}$ at the onset of the $v= \pm 2$ plateaus, where one edge state is almost perfectly transmitted, whereas the second edge state has a transmission close to $1 / 2$. This configuration corresponds precisely to the situation depicted in the original paper of McEuen et al., when only the upper LL of the filled conduction band is backscattered through the bulk. From Fig. 4, we find experimentally $R_{1(28), 37} \simeq 0.15 h / e^{2}$, $R_{1(28), 46} \simeq 0.05 h / e^{2}$, which is of the same order of magnitude than the maximal resistance values obtained from Fig. 7(c): $R_{1(28), 37} \simeq 0.04 h / e^{2}$ and $R_{1(28), 46} \simeq 0.02 h / e^{2}$. Therefore this simple model reproduces roughly the amplitudes of the measured magnetoresistances. Moreover, the model also predicts that $R_{1(28), 37}$ and $R_{1(28), 46}$ change sign when $B$ is reversed (or identically when the valence and conduction band are exchanged), in agreement with the experimental results of Fig. 4.

\section{APPENDIX B}

Figure 8 shows the nonlocal resistances $R_{28,37}(L / W=$ 1) and $R_{28,46}(L / W=2)$ measured for $G 14$, during one of the cool-downs at low temperature. The coefficient $\lambda_{\text {sh }}$ extracted from the fit can be higher than $200 \mu \mathrm{m}$, see panel (c). The $\theta_{\text {sh }}$ parameter given by the fit also has very high values. 
[1] S. Datta, Electronic Transport in Mesoscopic Systems (Cambridge University Press, England, 1997).

[2] P. L. McEuen, A. Szafer, C. A. Richter, B. W. Alphenaar, J. K. Jain, A. D. Stone, R. G. Wheeler, and R. N. Sacks, Phys. Rev. Lett. 64, 2062 (1990).

[3] A. K. Geim, Rev. Mod. Phys. 83, 851 (2011).

[4] D. Huertas-Hernando, F. Guinea, and A. Brataas, Phys. Rev. Lett. 103, 146801 (2009).

[5] N. Tombros, C. Jozsa, M. Popinciuc, H. T. Jonkman, and B. J. Van Wees, Nature (London) 448, 571 (2007).

[6] B. Dlubak, M.-B. Martin, C. Deranlot, B. Servet, S. Xavier, R. Mattana, M. Sprinkle, C. Berger, W. A. De Heer, F. Petroff et al., Nat. Phys. 8, 557 (2012).

[7] W. Yan, O. Txoperena, R. Llopis, H. Dery, L. E. Hueso, and F. Casanova, Nat. Commun. 7, 13372 (2016).

[8] D. Van Tuan, J. M. Marmolejo-Tejada, X. Waintal, B. K. Nikolić, S. O. Valenzuela, and S. Roche, Phys. Rev. Lett. 117, 176602 (2016).

[9] J. Balakrishnan, G. K. W. Koon, M. Jaiswal, A. C. Neto, and B. Özyilmaz, Nat. Phys. 9, 284 (2013).

[10] J. Balakrishnan, G. K. W. Koon, A. Avsar, Y. Ho, J. H. Lee, M. Jaiswal, S.-J. Baeck, J.-H. Ahn, A. Ferreira, M. A. Cazalilla et al., Nat. Commun. 5, 4748 (2014).

[11] J. Renard, M. Studer, and J. A. Folk, Phys. Rev. Lett. 112, 116601 (2014).

[12] R. Gorbachev, J. Song, G. Yu, A. Kretinin, F. Withers, Y. Cao, A. Mishchenko, I. Grigorieva, K. Novoselov, L. Levitov et al., Science 346, 448 (2014).

[13] Y. Wang, X. Cai, J. Reutt-Robey, and M. S. Fuhrer, Phys. Rev. B 92, 161411 (2015).

[14] A. A. Kaverzin and B. J. van Wees, Phys. Rev. B 91, 165412 (2015).

[15] D. A. Abanin, R. V. Gorbachev, K. S. Novoselov, A. K. Geim, and L. S. Levitov, Phys. Rev. Lett. 107, 096601 (2011).

[16] K. Gopinadhan, Y. J. Shin, R. Jalil, T. Venkatesan, A. K. Geim, A. H. C. Neto, and H. Yang, Nat. Commun. 6, 8337 (2015).

[17] D. A. Abanin, K. S. Novoselov, U. Zeitler, P. A. Lee, A. K. Geim, and L. S. Levitov, Phys. Rev. Lett. 98, 196806 (2007).

[18] M. Ribeiro, S. R. Power, S. Roche, L. E. Hueso, and F. Casanova, Nat. Commun. 8, 2198 (2017).

[19] G. M. Gusev, D. A. Kozlov, A. D. Levin, Z. D. Kvon, N. N. Mikhailov, and S. A. Dvoretsky, Phys. Rev. B 96, 045304 (2017).
[20] R. Ribeiro-Palau, F. Lafont, J. Brun-Picard, D. Kazazis, A. Michon, F. Cheynis, O. Couturaud, C. Consejo, B. Jouault, W. Poirier et al., Nat. Nanotechnol. 10, 965 (2015).

[21] T. J. B. M. Janssen, S. Rozhko, I. Antonov, A. Tzalenchuk, J. M. Williams, Z. Melhem, H. He, S. Lara-Avila, S. Kubatkin, and R. Yakimova, 2D Mater. 2, 035015 (2015).

[22] A. Nachawaty, M. Yang, W. Desrat, S. Nanot, B. Jabakhanji, D. Kazazis, R. Yakimova, A. Cresti, W. Escoffier, and B. Jouault, Phys. Rev. B 96, 075442 (2017).

[23] S. Lara-Avila, K. Moth-Poulsen, R. Yakimova, T. Bjørnholm, V. Fal'ko, A. Tzalenchuk, and S. Kubatkin, Adv. Mater. 23, 878 (2011).

[24] A. Lartsev, T. Yager, T. Bergsten, A. Tzalenchuk, T. J. B. M. Janssen, R. Yakimova, S. Lara-Avila, and S. Kubatkin, Appl. Phys. Lett. 105, 063106 (2014).

[25] J. Huang, J. Alexander-Webber, A. Baker, T. Janssen, A. Tzalenchuk, V. Antonov, T. Yager, S. Lara-Avila, S. Kubatkin, R. Yakimova et al., Phys. Rev. B 92, 075407 (2015).

[26] S. Kopylov, A. Tzalenchuk, S. Kubatkin, and V. I. Fal'ko, Appl. Phys. Lett. 97, 112109 (2010).

[27] L. J. van der Pauw, Philips Tech. Rev. 20, 220 (1958).

[28] D. A. Abanin, A. V. Shytov, L. S. Levitov, and B. I. Halperin, Phys. Rev. B 79, 035304 (2009).

[29] D. A. Abanin, S. V. Morozov, L. A. Ponomarenko, R. V. Gorbachev, A. S. Mayorov, M. I. Katsnelson, K. Watanabe, T. Taniguchi, K. S. Novoselov, L. S. Levitov et al., Science 332, 328 (2011).

[30] R. Delves, Rep. Prog. Phys. 28, 249 (1965).

[31] Y. M. Zuev, W. Chang, and P. Kim, Phys. Rev. Lett. 102, 096807 (2009).

[32] J. G. Checkelsky and N. P. Ong, Phys. Rev. B 80, 081413 (2009).

[33] P. Wei, W. Bao, Y. Pu, C. N. Lau, and J. Shi, Phys. Rev. Lett. 102, 166808 (2009).

[34] a. F. Young, J. D. Sanchez-Yamagishi, B. Hunt, S. H. Choi, K. Watanabe, T. Taniguchi, R. C. Ashoori, and P. Jarillo-Herrero, Nature (London) 505, 528 (2014).

[35] S. Slizovskiy and V. I. Fal'ko, Phys. Rev. B 97, 075404 (2018).

[36] P. G. Silvestrov and K. B. Efetov, Phys. Rev. B 77, 155436 (2008).

[37] E. Pop, V. Varshney, and A. K. Roy, MRS Bull. 37, 1273 (2012).

[38] M. Buttiker, IBM J. Res. Dev. 32, 317 (1988). 